\title{
El problema de la representación en la noción de verdad y su importancia para la comunicación social
}

\author{
Gabriel Zanotti \\ gabrielmises@yahoo.com
}

Facultad de Comunicación, Universidad Austral, Buenos Aires

Recibido: 28 de junio de 2017.

Aceptado: 9 de octubre de 2018 .

\section{Resumen}

En este artículo pretendemos dar una salida fenomenológica y escolástica al problema de la verdad como representación. Este afectará a la comunicación en general y, en particular, a la comunicación social, en tanto "representación" siga refiriendo a un supuesto mundo objetivo que olvida el mundo de la vida, esencial para una hermenéutica.

Palabras clave: verdad, representación, fenomenología, hermenéutica, mundo de la vida, signo formal.

\section{The problem of representation in the idea of truth} and its relevance in social communication

\begin{abstract}
In this article, we seek to provide an answer to the phenomenological and scholastic question of truth as representation. This will affect communication studies in general and, in particular, the field of social communications, as long as "representation" continues to refer to a supposedly objective world that forgets about the world of life, which is essential for hermeneutics.

Keywords: truth, representation, phenomenology, hermeneutics, the world of life, formal sign.

\section{O problema da representação na noção de verdade e sua importância para a comunicação social}

\section{Resumo}

Neste artigo pretendemos dar uma saída fenomenológica e escolástica ao problema da verdade como representação. Este afetará à comunicação em geral e, em particular, a comunicação social, enquanto "representação" continue referindo a um suposto mundo objetivo que esquece o mundo da vida, essencial para uma hermenêutica.
\end{abstract}

Palavras chave: verdade, representação, fenomenologia, hermenêutica, mundo da vida, signo formal. 
Gabriel Zanotti

El problema de la representación en la noción de verdad y su importancia para la comunicación social

\section{Introducción al problema}

En íntima conexión con el tema de la hermenéutica en comunicación social, ya abordado anteriormente (Zanotti, 2013) se encuentra la noción de verdad. Ya habíamos afirmado que la verdad, concebida como adecuación con los hechos, resulta en un principio muy atractiva para la objetividad que tantas veces se tiene como ideal en la comunicación social, pero habíamos visto que esa objetividad descansaba en un paradigma de la información en el que el sujeto cognoscente no tendría nada que ver con el diseño ni con los criterios de relevancia (agendas) que guían la redacción de los mensajes. Cuando se cae en la cuenta de que tal supuesto es imposible, la reacción es muchas veces la contraria: un relativismo en el que el sujeto, sencillamente, al diseñar su relato, impone sus propias categorías interpretativas sin relación con la realidad. La noción de verdad, en ese caso, se desploma porque se la había depositado en el banco de la información, que quiebra ante el redescubrimiento del sujeto por el giro hermenéutico.

Ya habíamos propuesto una salida en la afirmación de la realidad como intersubjetividad, el mundo de la vida, en el que los sujetos interactúan y habitan. De esa manera, estos no tienen que adecuarse a algo externo, sino que su hablar de su mundo de la vida es la expresión de ese mundo, con lo cual la verdad se re-define como la expresión de un mundo de la vida habitado, en el que el solo hablar según los juegos de lenguaje ya hay interpretación, interpretación de un horizonte vivido, cuyos mensajes serán más verdaderos cuanta mayor sea la radicación, la vivencia del sujeto en ese mundo, supuesta la sinceridad en su decir.

Sin embargo, queda un tema pendiente. Los sujetos se relacionan entre sí en una intersubjetividad en la que el cuerpo juega un papel esencial. Como tales, son ontológicamente diferentes uno de otro, y cualquiera podría decir que necesitamos aún la noción de re-presentación para decir que el otro no está en mí en tanto otro, sino en tanto una representación del otro en mí. Esto nos lleva a repasar nuevamente la historia del problema y a profundizar aún más en la noción de signo que tiene ese "estar en mí" del otro.

\section{Repaso del problema clásico de la representación}

Si la verdad es la adecuación de la inteligencia a la realidad, ¿cómo se produce esa adecuación? Heredando el típico planteo moderno acerca de que el conocimiento consiste en una relación de sujeto cognoscente a objeto conocido y suponiendo, a su vez, que el objeto no se subsume en el sujeto, surge la dificultad de cómo está seguro el sujeto de que conoce al objeto en cuanto real. El problema cartesiano casi se identifica con el problema del conocimiento. Si la respuesta consiste en decir que el sujeto tiene una idea que "corresponde" o "representa" al objeto (es decir, el objeto se hace presente 
en el sujeto), el inconveniente emerge nuevamente al preguntarnos si esa representación es una "verdadera" representación del objeto, con lo cual el problema se retrotrae hacia el infinito. Por supuesto, Descartes tuvo una manera "divina" de resolverlo: Dios, que se había demostrado a partir del sujeto, es el garante de que esa representación corresponde al mundo real creado por Él. Los racionalistas clásicos posteriores reelaboraron con mayor o menor originalidad ontológica el recurso a Dios como garante de que la representación no nos engaña. Malebranche sostuvo que cada vez que el sujeto se representa algo, Dios pone en él “la representación verdadera". Para Spinoza la diferencia ontológica entre sujeto y objeto desaparece porque hay una sola sustancia, identificada con lo divino en forma panteísta (antecedente de Hegel). Según Leibniz, cada idea corresponde a un objeto real por la armonía pre-establecida por Dios desde toda la eternidad.

Aún es objeto de debate si Descartes y los racionalistas posteriores reelaboraron de algún modo las tesis agustinistas de la verdad como participación en las Verdades Eternas en la mente de Dios (a favor, ver Leocata, 2012; en contra, ver Sciacca, 1954) pero asombra ante nuestros secularizados ojos actuales la recurrencia a Dios para solucionar el problema de la representación. Sin embargo, era una salida lógica. ¿De qué otro modo, si no con un garante infalible, podía asegurarse la certeza de un "tercero en discordia" (la idea), fruto de nuestras falibles facultades de conocimiento? Hume (1978) corta por lo sano y, al negar que se pueda demostrar la existencia de Dios, cae coherentemente en un escepticismo total.

Kant se da cuenta de la dificultad y por eso crea su propia síntesis, que intenta superar la dialéctica entre los racionalistas y Hume. Ya no hay cosa en mí que pondría en duda al objeto (una representación que no sabemos si representa al mundo) ni cosa en sí que no tenga que ver con el sujeto. Hay una proyección de nuestras categorías universales hacia la sensibilidad y, al juntarse, producen el conocimiento. La solución es muy buena, muy original, pero queda un sabor amargo: la famosa cosa en sí misma, la esencia de la cosa con independencia del sujeto, parece haber muerto para siempre $\mathrm{y}$, con ella, la noción tradicional de verdad.

Se podría decir que esto tiene dos reacciones. Una, la del racionalismo absoluto alemán: con Hegel, la cosa en sí se convierte en la "supercosa” en sí, el Espíritu Absoluto, en el que, al igual que para Spinoza, ya no hay problema de la representación porque sujeto y objeto se subsumen entre sí. La cuestión, en ese caso, es si se quiere asumir todos los supuestos ontológicos de Hegel.

La otra reacción fue la cientificista. A fines del siglo XIX, en un ambiente totalmente antihegeliano, el empirismo inglés siguió su curso y encontró en la metodología inductivista de las ciencias naturales, con Mill (1882) a la cabeza, la cosa en sí que se había perdido en el camino. Ahora la cosa en sí es el hecho, el dato, la ley científica "ob- 
jetiva", "probada" mediante el método experimental. El "sujeto" es el científico, el único ser humano que, al parecer, habría logrado librarse de sí mismo mediante un método que es, precisamente, "objetivo", y por eso lo libra a aquel de sí mismo y sus interpretaciones. Este paradigma tiene mucho éxito e instala en nuestra cultura la dicotomía objetivo-subjetivo, en tanto que la ciencia es ya la garantía de lo primero. Sí, así, Dios no se podría demostrar, por lo que tendría razón Hume, pero el resultado no tiene que ser el escepticismo: la ciencia es nuestra tabla de salvación porque ella no "representa". Por el contrario, es el único sistema de conocimiento que "presenta los hechos como son". Por lo tanto, la famosa definición de verdad como correspondencia se convierte en la correspondencia con los hechos y se hace "científica": si hay ciencia, hay verdad. La ciencia es ahora el garante. Por supuesto, esto se enfrenta luego con todo el giro epistemológico, conocido también como giro histórico de la filosofía de las ciencias (Koyré, 1979, 1980, 1988, 1994; Kuhn, 1985, 1989, 1996, 2000; Lakatos, 1989; Lakatos \& Musgrave, 1970; Feyerabend, 1981a, 1981b, 1989, 1991, 1992, 1995, 1999a, 1999b, 2001), pero no es este el momento de reseñarlo de nuevo (Zanotti, 2005).

El asunto central es que Heidegger, sin necesidad de debatir con la ciencia desde el lugar de esta, y habiendo afirmado ya que "el escándalo de la filosofía consiste en que esta demostración aún no haya sido hecha hasta ahora, sino, más bien, en que tales demostraciones sigan siendo esperadas e intentadas" (1998, p. 226), juega con esta noción de verdad en su famoso artículo "De la esencia de la verdad". Vale la pena citar el párrafo completo:

Hablamos de coincidencia dándole distintos significados. Por ejemplo, a la vista de dos monedas de cinco marcos que se encuentran sobre la mesa, decimos: las dos son iguales, coinciden. Ambas coinciden en su aspecto único. Tienen ese elemento en común y, por eso, desde ese punto de vista son iguales. Pero también hablamos de coincidir cuando, por ejemplo, afirmamos sobre una de las dos monedas de cinco marcos: esta moneda es redonda. Aquí, el enunciado coincide con la cosa. Ahora la relación ya no es entre cosa y cosa, sino entre un enunciado y una cosa. ¿Pero en qué pueden coincidir la cosa y el enunciado si los elementos que se han puesto en relación son distintos en lo tocante a su aspecto? La moneda es de metal. El enunciado no es nada material. La moneda es redonda. El enunciado no tiene para nada la naturaleza de algo espacial. Con la moneda se puede comprar algo. El enunciado sobre ella nunca puede ser un medio de pago. Pero, a pesar de toda esta desigualdad entre ambos, en la medida en que el enunciado es verdadero coincide con la moneda. Y, de acuerdo con el concepto corriente de verdad, ese modo de concordar tiene que ser una adecuación. ¿Pero cómo puede adecuarse a la moneda algo tan completamente desigual como el enunciado? Tendría que convertirse en moneda y de ese modo anularse a sí mismo por completo. Pero eso es algo que el enunciado no puede conseguir nunca. Si lo consiguiera, en ese mismo instante el enunciado ya no podría coincidir con la cosa en cuanto tal enunciado. Es la adecuación, el enunciado tiene que seguir siendo lo que es o incluso precisamente llegar a serlo. ¿En qué consiste su esencia, absolutamente distinta de cualquier cosa? ¿Cómo consigue el enunciado adecuarse a otro, a la cosa, permaneciendo y persistiendo precisamente en su esencia? (Heidegger, 2000, pp. 155-156). 
Austral Comunicación

Volumen 7 número 2 (diciembre de 2018): 251-262. ISSN 2313-9129

La objeción no podría estar mejor formulada. La proposición "la moneda es redonda", no es de metal, ni redonda, ni es un medio de pago, y sin embargo representa todo ello.

Por lo tanto, el problema de la representación subsiste. La ilusión de una ciencia "objetiva", libre del sujeto, fue refutada por Popper (1974, 1983, 1984, 1985a, 1985b, 1985c, 1985d, 1986, 1987, 1988, 1992, 1994, 1997, 1998, 1999), Popper y Lorenz (1990), Kuhn $(1985,1989,1996,2000)$, Lakatos (1989), Lakatos \& Musgrave (1970), Feyerabend (1981a, 1981b, 1989, 1991, 1992, 1995, 1999a, 1999b, 2001) y Koyré (1979, 1980, 1988, 1994), y está muy bien que así haya sido. No hay ciencia sin científico y su creatividad intelectual, sus conjeturas, sus paradigmas, sus acciones contrainductivas. Y tampoco se puede decir que cualquier proposición que nos parece verdadera lo sea porque ella misma se identifique con la cosa representada. ¿Cuál es, entonces, la solución?

\section{Un giro en la noción de realidad}

En los eternos debates sobre el idealismo o no idealismo de Husserl, pocas veces se advierte que este autor hace un giro ontológico esencial para las interpretaciones fenomenológicas de la hermenéutica (Leocata, 2003; Ricoeur, 2003, 2010). No es, por supuesto, el conocido giro en el "olvido del ser" de Heidegger, pero es un giro ontológico: lo real se identifica con la intersubjetividad. Ya no es cuestión de un objeto frente a un sujeto; se trata de un sujeto esencialmente en relación con otro: el mundo de la vida. Husserl sigue hablando de objetividad para destacar el carácter no psicologista del noema (Husserl, 1951, 1986). Sin embargo, se podría afirmar que la tradicional distinción sujeto-objeto de la filosofía postcartesiana en adelante, es superada con la noción de Leib, esto es, sujeto viviente, espiritual en el caso del ser humano, que "está en el mundo"; no en el sentido heideggeriano del término, sino en cuanto que el mundo de la vida, por un lado, es la realidad humana fundamental desde la cual se ve toda otra realidad (allí está el giro hermenéutico de la filosofía de Husserl); por otro, no es un objeto que el sujeto tiene delante: la persona vive en el mundo, está en él, "dentro de él”. El mundo no es un objeto externo sobre el que, por ende, pueda preguntarse si es real o no, porque la realidad del mundo de la vida es concomitante con la realidad de la persona que "está en" él. Y, por lo tanto, una conclusión fundamental: el mundo de la vida no tiene por qué ser "representado": está ya presente en el sujeto, que es inter-sujeto. Esta concepción, en ese sentido, supera el problema de la representación.

\section{El tema y el problema del signo formal}

Otro enfoque, asumido por algunos tomistas y neopragmaticistas (Nubiola \& Conesa, 1999; Llano, 1999; Peirce, 1998), ha sido recurrir a la noción de signo formal, 
que se identificaría con el signum quo de Santo Tomás (1967) y con el interpretantem de Pierce (1998). La noción de signo, a secas, coincide con la de signo instrumental. El signo es precisamente su papel de referir a otra cosa. Una flecha hacia la derecha colocada en un cartel en una ruta se identifica con su papel de indicarnos un giro a la derecha en la ruta.

Sin embargo, en principio, el signum quo de Santo Tomás no es eso: es "aquello por lo cual conocemos la cosa", es decir, lo que los escolásticos del siglo XVI identifican con el "concepto objetivo" (Maritain, 1976). Esto, como se sabe, tiene una profunda influencia en Frege, Bolzano y Brentano (Stadler, 2010), y finalmente en el noema de Husserl.

La ventaja que tiene el concepto de signo formal es que, contrariamente al signo instrumental, no representa a la cosa conocida. El signum quo es la misma cosa en tanto conocida. Esto es importante para evitar el problema de la representación en la intersubjetividad. Para ello, aclaremos algunos puntos.

En primer lugar, la realidad del otro sujeto no se asume solamente desde la intersubjetividad. Se da un paso ético adicional: el compromiso existencial con el otro. Así, lo que coloca al sujeto en la certeza de la realidad del otro no es solo la noción misma de otredad, sino una situación en la que éticamente ha salido de la razón instrumental para advertir al otro como un "tú" en el sentido de Buber (1994). Condiciones para esta posición ética son la situación límite de Jaspers (1978), la existencia auténtica de Heidegger (1998) y el rostro sufriente del otro de Lévinas (1991).

En segundo lugar, una vez que Pedro está ética y filosóficamente seguro de que conoce a Juan en tanto Juan (en tanto otro), puede advertir a la vez que una cosa es Juan en tanto Juan y otra Juan en tanto conocido. Juan en tanto conocido se identificaría con el concepto objetivo que Pedro tiene de Juan. Ese "Juan en tanto conocido" no está en el medio de Pedro y Juan: está "por encima"; no es lo conocido: es aquello por lo cual conocemos.

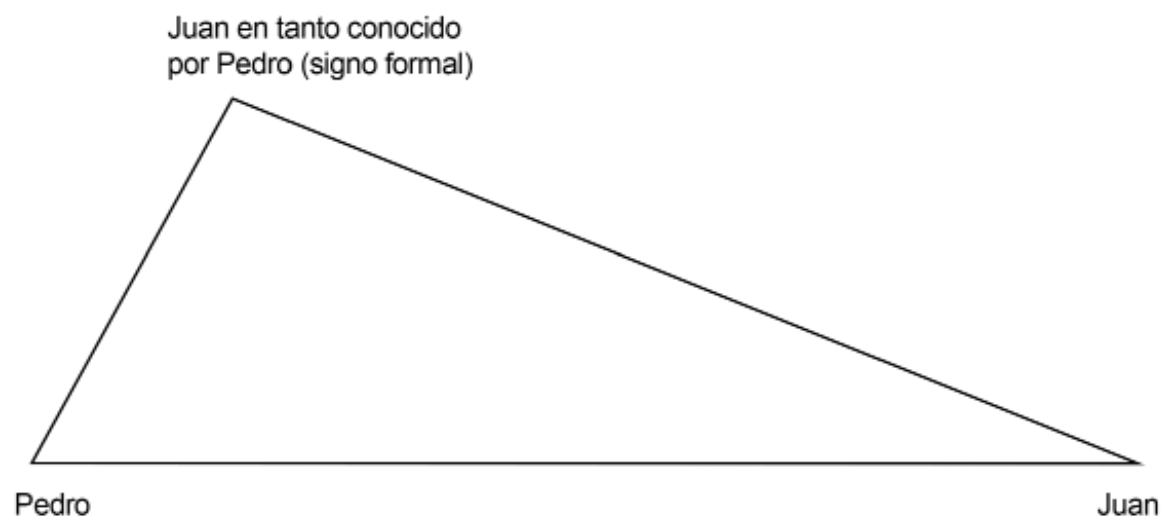

Figura 1. Función del signo formal. 
Austral Cmunicación

Volumen 7 número 2 (diciembre de 2018): 251-262. ISSN 2313-9129

El esquema es nuestro, pero coincide levemente con el famoso esquema triádico de Pierce (1998), en el que el signo se identifica más bien con la idea de interpretantem.

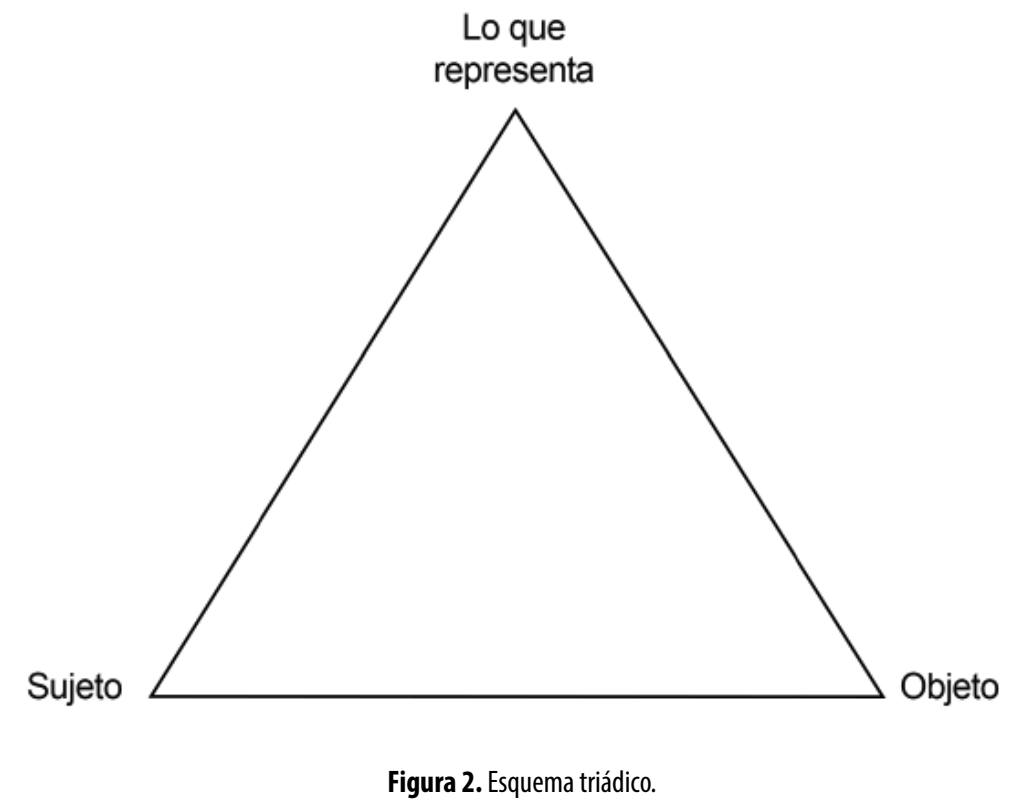

A su vez, la verdad sobre Juan (por ejemplo, "es verdad que Juan es profesor de filosofía") implica una reflexión que el sujeto hace sobre la proposición "Juan es profesor de filosofía" (Santo Tomás de Aquino, 1986). Por esta razón, la expresión "es verdad que" es metalingüística con respecto a "Juan es profesor de filosofía" (Llano, 1979). Esa reflexión supone la inmaterialidad del concepto objetivo, dado que los sentidos no pueden re-flexionar sobre sí mismos. Por ende, es obvio que el concepto objetivo de moneda en tanto concepto objetivo no se identifica con algo material singular.

De esta manera, podemos volver al ejemplo de la moneda: por supuesto, la proposición "la moneda es redonda" no se identifica con la moneda, y por ello no es de metal, ni redonda, ni medio de cambio. Pero la verdad de la proposición no implica que la realidad de la moneda sea un objeto más allá del sujeto. Implica que lo que sea moneda es tal desde un mundo de la vida que es interno al sujeto. Lo mismo -y más aún-con respecto a los otros conocidos en tanto otros: Juan no es un objeto con respecto a Pedro; es otro sujeto conocido en el mundo de la vida. Por lo demás, Pedro no es una idea de Juan, cuya realidad hay que demostrar (allí la idea sería signo instrumental). Antes bien, Pedro en tanto conocido por Juan es el mismo Pedro en tanto conocido, un signum quo, no un signum quod. No hay una idea de Pedro que sea un tercero en 
Gabriel Zanotti

El problema de la representación en la noción de verdad y su importancia para la comunicación social

discordia entre Pedro y Juan (es decir, Pedro, idea "objeto" de Juan, y Juan), sino que hay dos, Pedro y Juan, y ambos "en tanto conocidos" por uno y otro recíprocamente.

Finalmente, una objeción que se puede hacer a lo anterior es si esta solución del signo formal no entrañaría asumir nuevamente una tesis agustinista del conocimiento. Esto es, que toda verdad que el ser humano pueda pronunciar implica la participación en el intelecto divino, donde están las verdades en sí. Ello sería a su vez una forma de entender a Descartes. Nos mantenemos abiertos a tal objeción, advirtiendo solamente que esto supondría un replanteo de toda la teoría de la verdad desde la armonía entre razón y fe, tema que excede los objetivos de este humilde artículo.

\section{La importancia para comunicación social}

Todo lo tratado hasta aquí es fundamental para comunicación social porque la verdad de una proposición ya no tiene que ver con una noción fáctica del hecho, en la que la interpretación no interviene. Por el contrario, la verdad no es más que la expresión del mundo de la vida habitado por el sujeto. La mayor radicación en ese mundo de la vida implica una mayor verdad. Cuando Borges habla de literatura inglesa, es el "estar en el mundo de" esa literatura lo que comporta una mayor verdad en las proposiciones de Borges y, por ende, su mayor interpretación. De ese modo, Borges es eslabón de sentido entre su mundo (la literatura inglesa) y su audiencia. En cuanto tal, no opera como un eslabón de mayor distancia entre el emisor y esta, sino al revés: el emisor comunica, pone en común, su mundo con el de la audiencia, siempre y cuando habite su mundo y, a la vez, el de ella.

Lo que ocurre es que estas mediaciones hermenéuticas se dan por obvias, en actitud natural, o se las niega desde la contraposición positivista sujeto/hechos. Se teme que las mediaciones hermenéuticas de los sujetos disminuyan o anulen la verdad, cuando hemos visto que es al revés. Ahora bien, que esas mediaciones hermenéuticas implican tomas de posición sobre el mundo -o, en otros términos, horizontes de pre-comprensión- resulta obvio: el comunicador debe estar formado, por ende, en la defensa de la verdad de su horizonte más que en la constatación fáctica de "hechos" que, como tales, sin horizontes, no existen en la comunicación social.

El debate de la verdad de los horizontes no conlleva volver a supuestos hechos que los justifiquen; antes bien, remite a posiciones filosóficas últimas que están implícitas en aquellos. En ciencias sociales y en los debates ideológicos (aunque no sean lo mismo), lo que siempre se encuentra de fondo es una determinada concepción de antropología filosófica. Un liberal clásico y un marxista, si quieren debatir en serio, no tienen que ir a presuntos índices que manejan los economistas de coyuntura. Detrás de la teoría de la explotación del marxista está la dialéctica hegeliana; detrás de la defensa del mercado del liberal clásico hay una concepción de antropología filosófica según la 
cual el ser humano es individuo, con explicaciones sobre lo individual que se remontan a autores como Menger, Smith y corrientes como la Segunda Escolástica.

En ciencias naturales sucede otro tanto. Detrás de las discusiones sobre las interpretaciones idealistas o realistas de la física cuántica, se encuentra una "filosofía de la física" que no tiene que ver con experimentos, sino con debates cosmológicos y ontológicos tales como si el universo es determinado o indeterminado, y si el indeterminismo es del universo en sí mismo o del sujeto que lo entiende. El físico que desee resolver esas controversias debe saber que ha de remontarse a la filosofía de la naturaleza si quiere defender la verdad de su interpretación de la física cuántica.

Esto tiene una fundamental importancia a la hora de analizar el caso de las fake news. Por ejemplo, no hay duda de que, con respecto a la situación económica actual de los Estados Unidos, ${ }^{1}$ Fox News y The New York Times tienen una visión distinta. Los meros "datos" (como si pudiera haberlos) no solucionan la cuestión, porque ambos medios deben reconocer que la desocupación ha bajado y que hay un booming en la situación económica. El asunto es por qué. Y allí se ponen en juego los horizontes. La desocupación bajó, pero ello puede deberse a una mayor protección arancelaria y a un mayor control de inmigración, y ninguna de las dos cosas implica un aumento en la productividad global. Desde ese horizonte, la interpretación de la baja de la desocupación responde tanto a una mayor tasa de inversiones como a medidas intervencionistas contrarias al mercado libre defendido por Fox News. Pero en ese caso, como vemos, hay que estar dispuesto a defender la verdad de ese horizonte. Los meros facts, sin horizontes, no existen.

Por lo tanto, hay que estar atentos a si los actuales debates sobre la "postverdad" no tienen implícita una noción positivista de verdad, en la que los medios tradicionales señalan "hechos sin horizontes" y las redes sociales, en cambio, "interpretan", lo que produce la "postverdad". Si ese es el planteo, está viciado filosóficamente ab initio por el positivismo. Todo medio de comunicación interpreta. La mentira es un tema ético, que presupone la posibilidad de decir la verdad, y esta, como hemos visto, más se dice cuanto más puede interpretar el emisor del mensaje el mundo que habita.

Más allá de la "mentira" como obvia definición ética de fake news, las diversas posiciones ideológicas se acusan mutuamente de afirmarlas, como si una posición tuviera los facts y la otra, la interpretación. Esto es un error. Por ejemplo, en abril de 1982, ¿cómo eran los titulares en Inglaterra y Argentina? En Inglaterra, se hablaba de invasion. En Argentina, de recuperación. ¿Cuál era allí la fake news? Y si un título dice "XX interrumpió su embarazo" y otro afirma, de la misma XX, "asesinó a su hijo", ¿cuál es allí la fake news? ¿Quién está "mintiendo"? Como vemos, estas cosas no tienen solución, excepto que los comunicadores y la audiencia tomen conciencia de sus propios

Nos referimos al aumento de la ocupación en los primeros dos años de la actual presidencia de Donald Trump. 
Gabriel Zanotti

El problema de la representación en la noción de verdad y su importancia para la comunicación social

horizontes y estén dispuestos a debatir la verdad de ellos, pero renunciando a "facts $\sin$ horizontes" como clave de la verdad de su posición.

Finalmente, la verdad no puede desvincularse de su sentido ético. La sinceridad como condición de diálogo (conformidad entre lo que se dice y lo que se piensa) es la condición de la credibilidad del mensaje, es decir, que el mensaje sea creíble sobre todo para aquellos que habitan otros mundos. Esto, para la comunicación y, en especial, la comunicación social, es esencial: es el capital profesional del comunicador, la credibilidad de su "firma". No garantiza la infalibilidad, pero sí la honestidad y la ética fundamental de algunos "no": no entrar en la banalidad del chisme, no calumniar, no atentar contra la intimidad de los demás. Y también respalda los "sí": comunicar mundos; acercar horizontes; ejercer la conciencia crítica sobre la base del amor a la justicia, pero nunca a partir de la venganza o la ideología. El comunicador debe poner en común: tender puentes, evitar malentendidos y buscar lo universal.

\section{Referencias}

Buber, M. (1994). Yo y tú. Buenos Aires: Nueva Visión.

Feyerabend, P. (1981a). Philosphical Papers (1 y 2), Cambridge/Nueva York: Cambridge University Press.

Feyerabend, P. (1981b). Tratado contra el método. Madrid: Tecnos.

Feyerabend, P. (1989). Diálogo sobre el método. Madrid: Cátedra.

Feyerabend, P. (1991). Diálogos sobre el conocimiento. Madrid: Cátedra.

Feyerabend, P. (1992). Adiós a la razón. Madrid: Tecnos.

Feyerabend, P. (1995). Killing Time. Chicago/Londres: University of Chicago Press.

Feyerabend, P. (1999a). Ambigüedad y armonía. Barcelona: Paidós.

Feyerabend, P. (1999b). Knowledge, Science and Relativism. Philosophical Papers, vol. 3.

Chicago/Nueva York: Cambridge University Press.

Feyerabend, P. (2001). La conquista de la abundancia. Barcelona: Paidós.

Heidegger, M. (1998). Ser y tiempo. Santiago de Chile: Editorial Universitaria.

Heidegger, M. (2000). De la esencia de la verdad. En: Hitos (pp. 151-171). Madrid:

Alianza.

Hume, D. (1978). A Treatise of Human Nature. Oxford: Oxford University Press.

Husserl, E. (1951). La filosofía como ciencia estricta. Buenos Aires: Universidad de

Buenos Aires.

Husserl, E. (1986). Ideas relativas a una fenomenología pura y una filosofía fenomenológica. México/Buenos Aires: Fondo de Cultura Económica.

Jaspers, K. (1978). La filosofía. México/Buenos Aires: Fondo de Cultura Económica.

Koyré, A. (1979). Del mundo cerrado al universo infinito. Buenos Aires: Siglo XXI.

Koyré, A. (1980). Estudios galileanos. Buenos Aires: Siglo XXI. 
Austral Comunicación

Volumen 7 número 2 (diciembre de 2018): 251-262. ISSN 2313-9129

Koyré, A. (1988). Estudios de historia del pensamiento científico. Buenos Aires: Siglo XXI.

Koyré, A. (1994). Pensar la ciencia. Barcelona: Paidós.

Kuhn, T. S. (1971). La estructura de las revoluciones científicas. México: Fondo de Cultura Económica.

Kuhn, T. S. (1985). La revolución copernicana. Madrid: Orbis.

Kuhn, T. S. (1989). ¿Qué son las revoluciones científicas? y otros ensayos. Barcelona: Paidós.

Kuhn, T. S. (1996). La tensión esencial. México: Fondo de Cultura Económica.

Kuhn, T. S. (2000). The Road since Structure. Chicago/Londres: University of Chicago Press.

Lakatos, I. (1989). La metodología de los programas de investigación científica. Madrid: Alianza Editorial.

Lakatos, I. \& Musgrave, A. (eds.) (1970). Criticism and the Growth of Knowledge. Chicago/Londres: Cambridge University Press.

Leocata, F. (2003). Persona, lenguaje, realidad. Buenos Aires: Educa.

Leocata, F. (2012). Del Iluminismo a nuestros días. Buenos Aires: Biblioteca Nacional del Bicentenario.

Lévinas, E. (1991). Ética e infinito. Madrid: Visor Distribuciones.

Llano, A. (1979). Metafísica y lenguaje. Pamplona: Eunsa.

Llano, A. (1999). El enigma de la representación. Madrid: Síntesis.

Maritain, J. (1976). El orden de los conceptos. Buenos Aires: Club de Lectores.

Mill, J. S. (1882). A System of Logic, Ratiocinative and Inductive. Nueva York: Harper \& Brothers Publishers.

Nubiola, J. \& Conesa, F. (1999). Filosofía del lenguaje. Barcelona: Herder.

Peirce, C. (1998). The essential Peirce, vol. 2. Bloomington: Indiana University Press.

Popper, K. (1974). Replies to my Critics. Illinois: Arthur Schilpp Lasalle.

Popper, K. (1983). Conjeturas y refutaciones. Barcelona: Paidós.

Popper, K. (1984): Sociedad abierta; universo abierto. Madrid: Tecnos.

Popper, K. (1985a). Realismo y el objetivo de la ciencia. Madrid: Tecnos.

Popper, K. (1985b). La lógica de la investigación científica. Madrid: Tecnos.

Popper, K. (1985c). Búsqueda sin término. Madrid: Tecnos.

Popper, K. (1985d). Teoría cuántica y el cisma en Física. Madrid: Tecnos.

Popper, K. (1986). El universo abierto. Madrid: Tecnos.

Popper K. (1987). La miseria del historicismo. Madrid: Alianza Editorial.

Popper, K. (1988). Conocimiento objetivo. Madrid: Tecnos.

Popper, K. (1992). Un mundo de propensiones. Madrid: Tecnos.

Popper, K. (1994). The Myth of the Framework. Londres/NuevaYork: Routledge. 
Gabriel Zanotti

El problema de la representación en la noción de verdad y su importancia para la comunicación social

Popper, K. (1997). El cuerpo y la mente. Barcelona: Paidós.

Popper, K. (1998). The World of Parmenides. Londres/Nueva York: Routledge.

Popper, K. (1999). All Life is Problem Solving. Londres/Nueva York: Routledge.

Popper, K. \& Lorenz, K. (1990). O futuro esta aberto. Lisboa: Fragmentos.

Ricoeur, P. (2003). El conflicto de las interpretaciones. México/Buenos Aires: Fondo de Cultura Económica.

Ricoeur, P. (2010). Del texto a la acción. México/Buenos Aires: Fondo de Cultura Económica.

Santo Tomás de Aquino. (1986). Quaestio disputata de veritate. Turín: Marietti.

Santo Tomás de Aquino. (1967). Suma contra gentiles. Madrid: Biblioteca de Autores Cristianos.

Sciacca, M. F. (1954). Historia de la filosofía. Barcelona: Luis Miracle.

Stadler, F. (2010). El Círculo de Viena. México/Buenos Aires: Fondo de Cultura Económica.

Zanotti, G. (2005). Hacia una hermenéutica realista. Buenos Aires: Universidad Austral.

Zanotti, G. (2013). La llamada objetividad de los medios de comunicación y sus paradójicas consecuencias para la verdad. Austral Comunicación 2(1), 27-41.

El autor agradece a su ayudante alumna María Cecilia Muratore por su ayuda en la elaboración de las referencias. 